\title{
Electrical discharge machining studies on reactive sintered FeAl
}

\author{
A K KHANRA*, S PATRA ${ }^{\dagger}$ and M M GODKHINDI \\ Department of Metallurgical and Materials Engineering, ${ }^{\dagger}$ Central Workshop and Instrument Service, Indian Institute \\ of Technology, Kharagpur 721 302, India
}

MS received 7 November 2005

\begin{abstract}
Electrical discharge machining (EDM) studies on reactive sintered FeAl were carried out with different process parameters. The metal removal rate and tool removal rate were found to increase with the applied pulse on-time. The surface roughness of machined surface also changed with the applied pulse on-time. XRD analysis of machined surface of sintered $\mathrm{FeAl}$ showed the formation of $\mathrm{Fe}_{3} \mathrm{C}$ phase during the EDM process. The debris analysis was used to identify the material removal mechanism occurring during the EDM of sintered FeAl.
\end{abstract}

Keywords. FeAl; sintering; EDM.

\section{Introduction}

Electrical discharge machining (EDM) is one of the most widely used non-conventional machining processes. The EDM technique was developed in 1943 by Lazarenko and Lazarenko of Moscow University. Then onwards it has been the widely accepted standard process for manufacturing of dies for forging/extrusion, machining of super alloys, composites, ceramics etc (Ho and Newman 2003; Singh et al 2004). In EDM, the tool and the workpiece are connected to two electrodes and they are separated by a dielectric fluid. At the beginning of EDM operation, a high voltage is applied across the narrow gap of typically about $20-300 \mu \mathrm{m}$ (depending on current) between the two electrodes. The high voltage induces an electric field in the insulating dielectric that is present in the narrow gap. This causes conducting particles suspended in the dielectric to concentrate at the points of the strongest electric field, which in turn results in a bridge being formed between the electrodes. At the same time, negatively charged particles are emitted from the cathode and collide with neutral particles in the gap between the electrodes, forming electrons and positively charged particles. This dielectric breakdown process spreads at an explosive rate, resulting in the formation of a conducting channel between the electrodes, called plasma channel. The energy input in $\mathrm{EDM}(P=V I t$ directly depends on discharge voltage, current and pulse on-time (Norasetthekul et al 1999). This channel conducts electricity between two electrodes, the voltage $(V)$ drops within a few milliseconds and current increases. The channel grows with time called pulse on-time $(t)$ which is set by operator. This channel

\footnotetext{
*Author for correspondence (asit_iitkgp@yahoo.com)
}

creates a high temperature generation. At this condition localized evaporation of electrodes takes place in the plasma channel. Wire cut and die sinking are the two types of EDM machine (Cheng et al 1996).

Intermetallic alloys such as $\mathrm{FeAl}$ are potential materials for high temperature applications. This iron aluminide has a remarkable oxidation and corrosion resistance. Its main applications are in hot gas filters, furnace fixtures, heating elements, automobile components subjected to high temperature etc (Yamaguchi et al 2000). Iron aluminide parts are currently produced by various liquid melt routes but poor machinability puts considerable limitation (Prasad et al 2001). The EDM technique which has been found suitable in case of some ceramic materials (with a maximum resistivity of $0.17 \mu . \mathrm{m})$ could be applicable in case of inte rmetallics (Gadalla et al 1991).

In the present investigation, $\mathrm{FeAl}$ was produced by reactive sintering and its EDM (die sinking type) behaviour was investigated with different process variables. The composition analysis of the machined surfaces and mechanism of material removal were also investigated.

\section{Experimental}

FeAl bars were produced by reactive sintering of stoichiometric mixture of $\mathrm{Fe}$ and $\mathrm{Al}$ powders at $1273 \mathrm{~K}$ in a tubular silicon carbide resistance furnace with a continuous flow of hydrogen gas. The sintered FeAl showed $94 \%$ of theoretical density. XRD analysis showed the presence of $\mathrm{FeAl}$ as a major phase and $\mathrm{Fe}_{3} \mathrm{Al}$ as a minor phase. The Vickers hardness was found to be $345 \mathrm{~kg} / \mathrm{mm}^{2}$ (load at $25 \mathrm{~g}$ and loading time, $1.5 \mathrm{~s}$ ).

EDM of sintered FeAl bar was carried out by Charmilles ELERODA 10 machine. Copper electrode (99.5\% 
pure) and BP cutting oil were used as tool material and dielectric liquid, respectively. The EDM study was carried out for different variables such as metal removal rate with

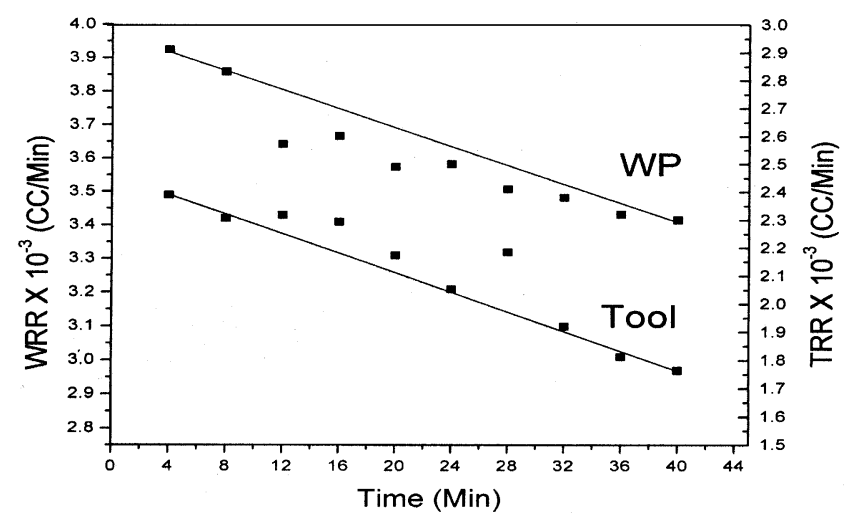

Figure 1. Removal rate of WP and tool when tool and WP with time.

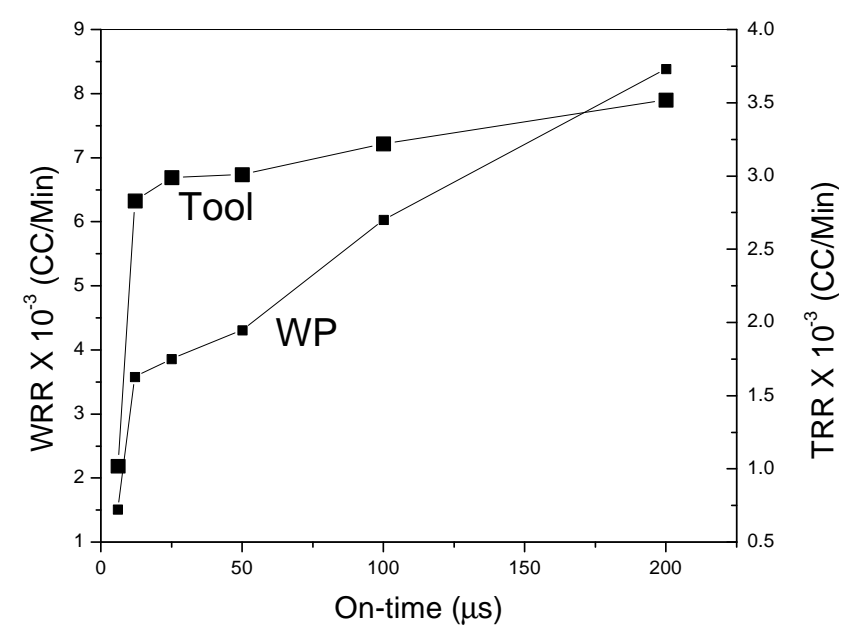

Figure 2. Removal rate of WP and tool with different on-times.

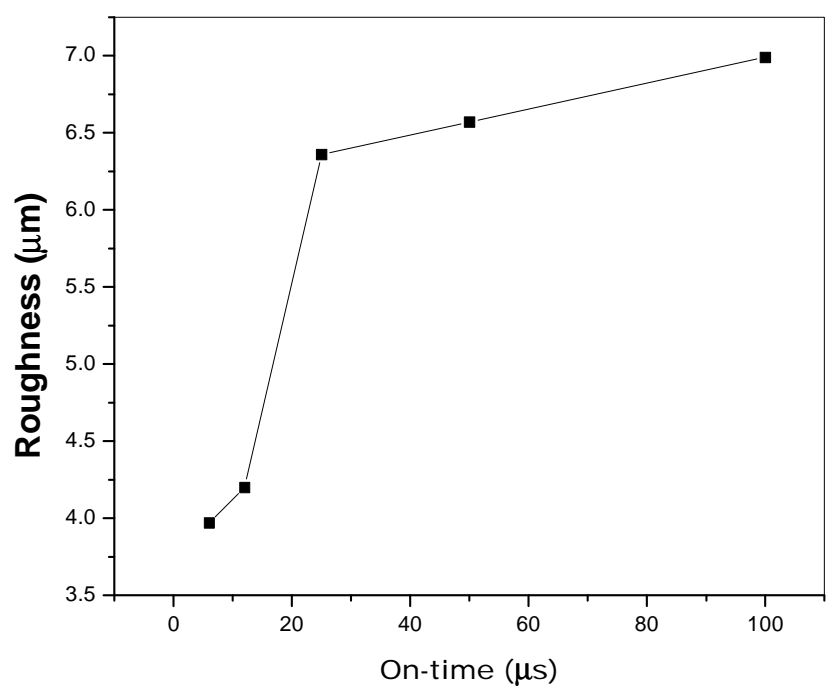

Figure 3. Surface roughness of WP as a function of on-time. time, pulse on-time etc. Surface roughness on the machined surface was also investigated as a function of pulse ontime. The entire EDM study was carried out at $10 \mathrm{amp}$ current and $45 \mathrm{~V}$ gap voltage. The microstructure of the tool and workpiece surface was studied by scanning electron microscopy (SEM) (JEOL, JSM 840A). The phase analysis of machined surfaces was carried out by XRD (Philips 1840) technique using iron filtered $\mathrm{CoK}_{\alpha}$ radiation $(\lambda=1.7889 \AA)$. The chemical composition on the EDM surfaces was also analysed by SEM/EDS. The debris was carefully collected after the EDM (pulse of-time was $25 \mu \mathrm{s}$ ) and dried. The morphology of the debris particles and composition were investigated by SEM and SEM/ EDS, respectively.

\section{Results and discussion}

During EDM the high temperature generation results in continuous wear on both the surface of tool and workpiece. Figure 1 indicates a linear relationship with time for both the workpiece removal rate (WRR) and tool removal rate (TRR) (when WP and tool materials were cathode and anode, respectively). The metal removal rate (WRR) decreases with time. During EDM, a continuous burning of cutting fluid takes place which would give out a carbon residue. The visual black layer was found on machined surface, which could decrease the WRR.

The WRR increased with the pulse on-time (figure 2). Initially the rate increased drastically with the increase of pulse on-time and later a slow increase of WRR was found. The increase of WRR with pulse on-time is expected because the input power was increased. The increase of WRR might be due to flow of more number of positive ions from tool to WP which causes more craters on the workpiece. Due to rapid metal removal of WP with increasing pulse on-time, the surface roughness increased drastically (figure 3). The surface roughness of WP increased suddenly from pulse on-time, 12-25 $\mu$ s and increased linearly afterwards. A similar phenomenon was also found in case of tool surface. But the roughness of tool was much less than that of the WP. It has been further noticed that by changing polarity the WRR decreases.

The surface morphology of the tool and the workpiece is shown in figure 4 . The surface morphology showed rough surface with non-uniform distribution of spherical and nonspherical agglomerates. SEM/EDS composition analysis of the tool surface showed the presence of $\mathrm{C}, \mathrm{Fe}$ and $\mathrm{Al}$ (figure 5). This carbon and wear materials (coming from tool and workpiece) were flushed out by a continuous flow of the dielectric fluid. But some part of materials was deposited on the surface of the tool and workpiece. Similarly, presence of $\mathrm{C}$ and $\mathrm{Cu}$ was found on the workpiece surface. It means that during EDM, a significant amount of workpiece material was found to transfer from workpiece surface to tool surface and some amount of material was transferred from tool to the workpiece. 

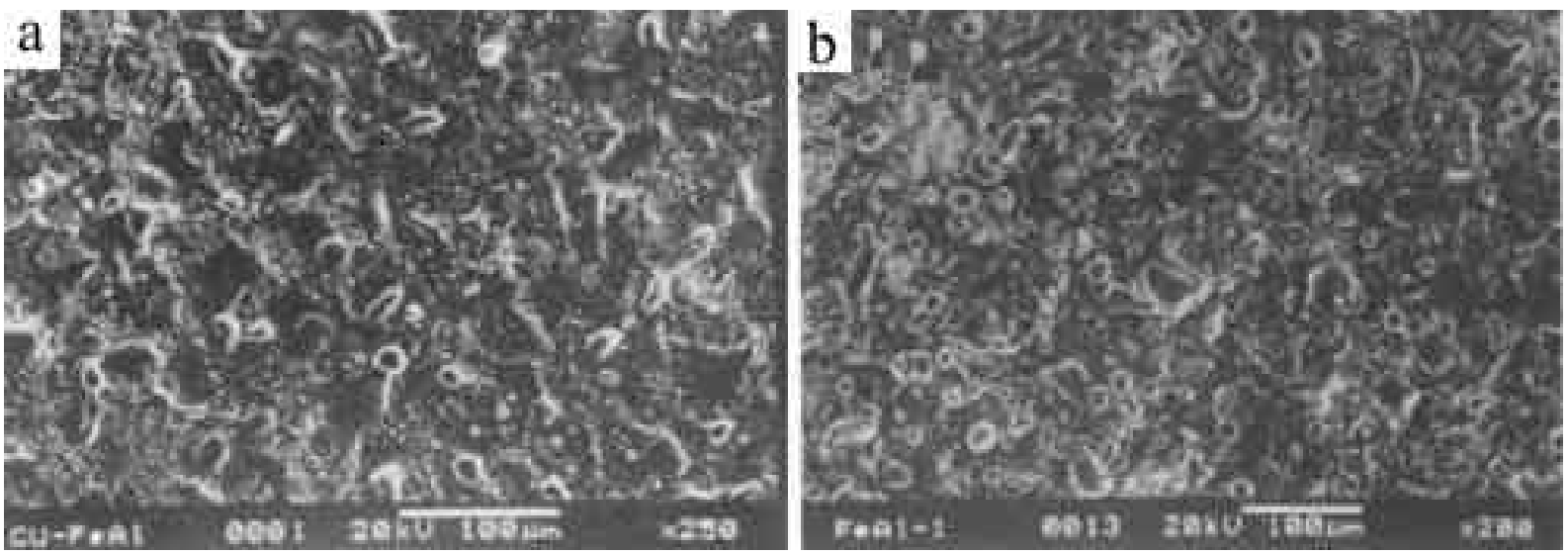

Figure 4. SEM images of machined surface: a. tool and b. workpiece.

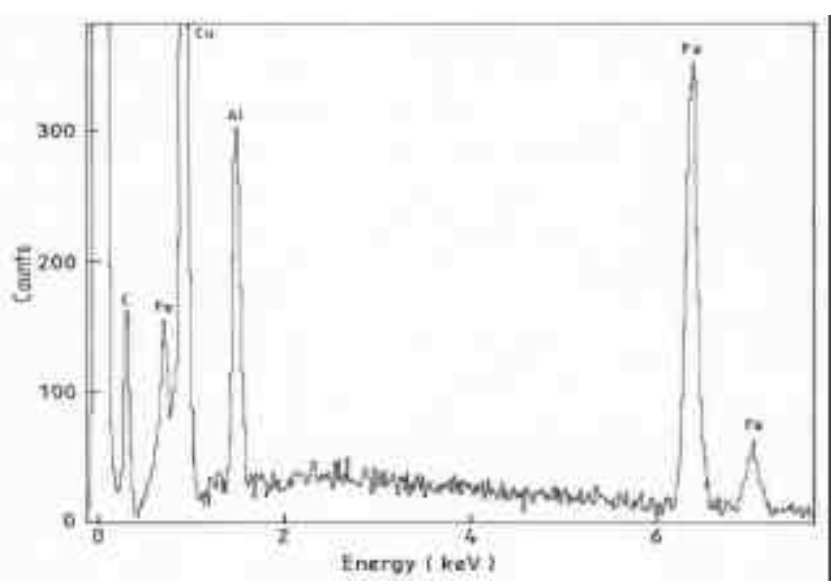

Figure 5. EDS spectrum of tool after machining.

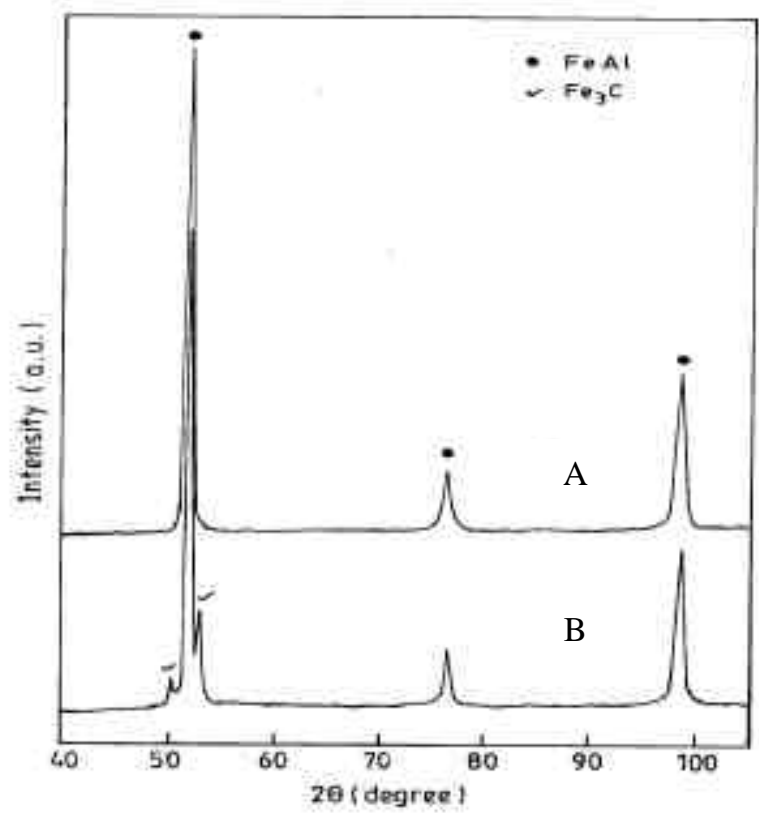

Figure 6. XRD pattern of workpiece (FeAl): A. before EDM and B. after EDM.
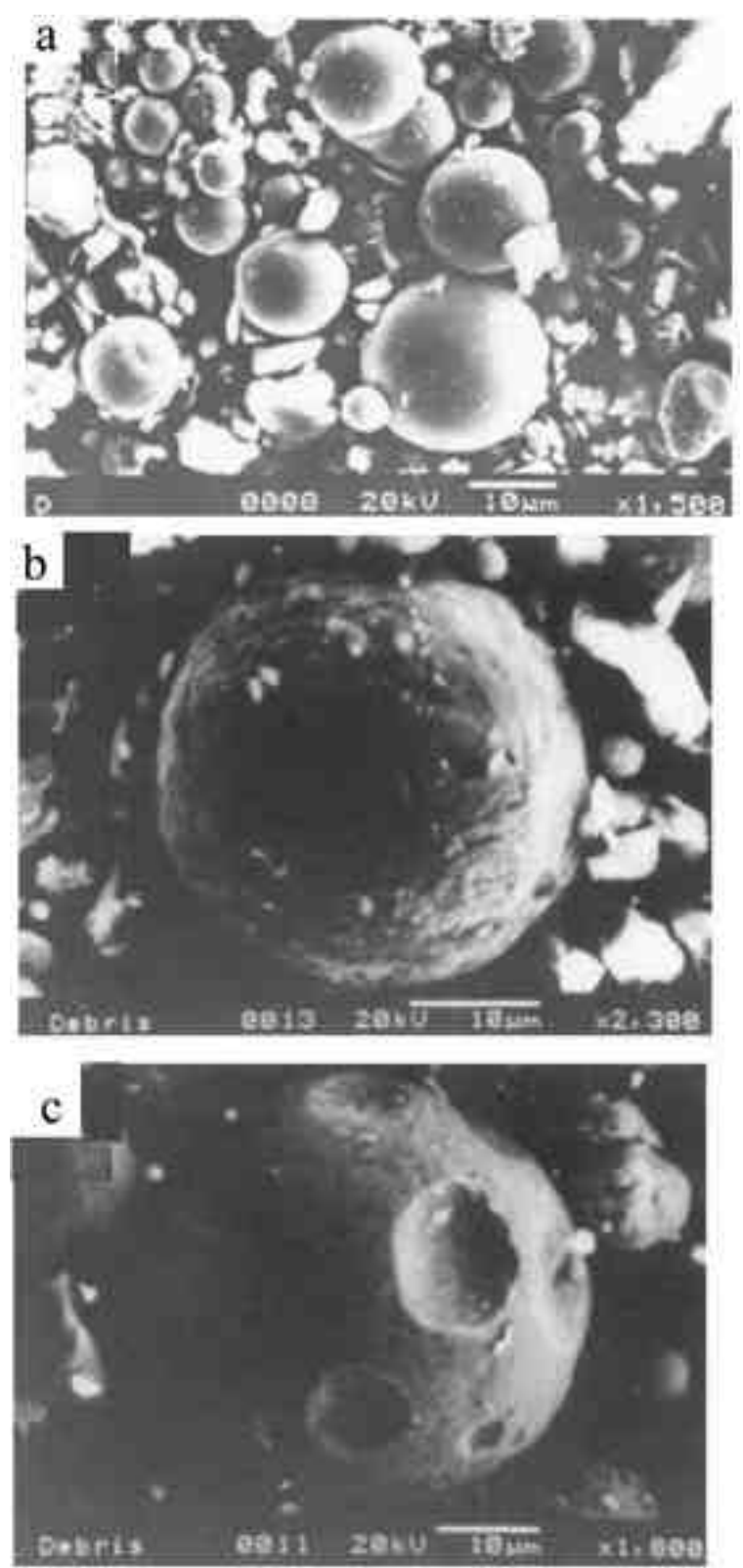

Figure 7. SEM images of a. debris, b. debris with heterogeneous nucleation and c. debris with dent formation. 


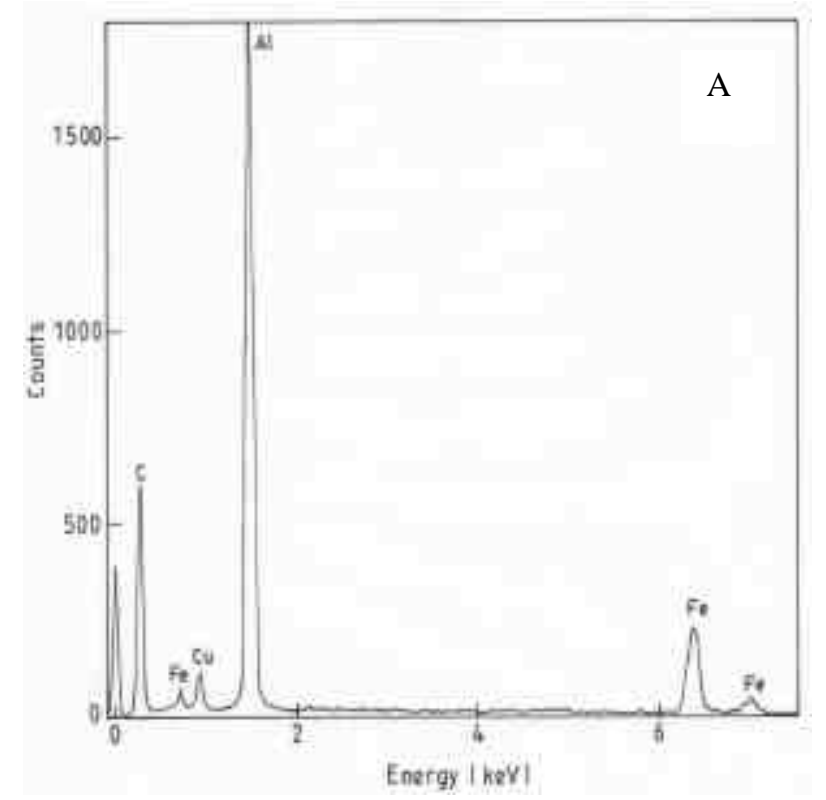

Figure 8. EDS of A. spherical and B. non spherical particles.

Formation of $\mathrm{Fe}_{3} \mathrm{C}$ phase was found on the machined surface of the workpiece (figure 6). During EDM the WP reacts with carbon residue to form $\mathrm{Fe}_{3} \mathrm{C}$. No phase change was found on the tool surface.

SEM images of the debris are shown in figure 7. The debris (figure 7a) mainly consisted of spherical and non spherical particles. The EDS analysis of spherical particle (figure 8a) showed the presence of mainly $\mathrm{Fe}, \mathrm{Al}$ and $\mathrm{C}$ with $\mathrm{Cu}$ as a trace element. This indicated that spherical particle formation was mainly due to solidification of wear material of WP. The presence of spherical particle indicated that the material removal mechanism was due to the melting and evaporation. The EDS analysis of non spherical particle showed (figure $8 \mathrm{~b}$ ) the presence of $\mathrm{Cu}$, $\mathrm{Fe}, \mathrm{Al}$ and C. During EDM the generation of high thermal stress leads to generation of micro cracks on the machined surfaces. Under severe cases, the materials come out layer by layer i.e. irregular shape. This indicated that the thermal spalling was also another material removal mechanism. But the presence of spherical shape particles in large numbers indicated that the melting and evaporation mechanism was dominating in the present study. The thermal spalling mechanism could dominate when input power is more. Figure $7 \mathrm{a}$ also shows formation of satellite, which was due to tiny particle (resulting from spark) solidified over the surface of a bigger particle. During EDM typical heterogeneous nucleation was found (figure $7 b$ ). Presence of impurities like $\mathrm{C}, \mathrm{Cu}$ etc acts as nucleation sites for heterogeneous nucleation of WP. This indicated that the debris particles were not amorphous in nature. The collision of debris particles during EDM led to dent formation (figure 7c).

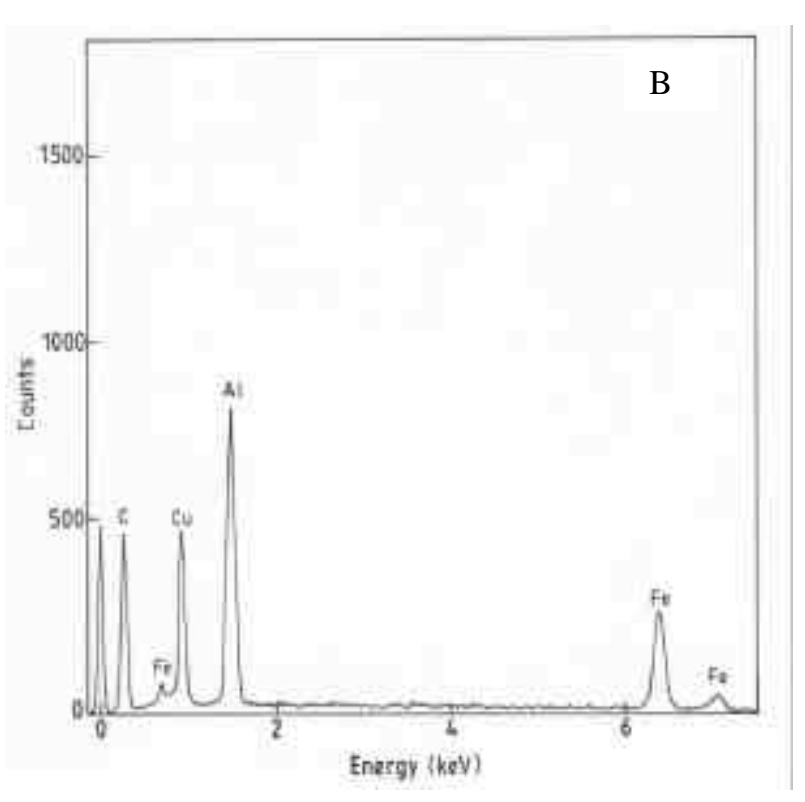

\section{Conclusions}

The present study shows that the intermetallic FeAl can be machined by EDM. Formation of $\mathrm{Fe}_{3} \mathrm{C}$ phase was found during the EDM process. The material removal mechanism was mainly due to melting and evaporation. The debris micrographs showed formation of satellites, heterogeneous nucleation and dent formation.

\section{Acknowledgements}

The authors are thankful to B P Mirdha, R Maity and A Ghosh, IIT Kharagpur, for help in carrying out XRD, SEM/EDS and surface roughness measurements.

\section{References}

Cheng Y M, Eubank P T and Gadalla A M 1996 Mats and Manuf. Proc. 11565

Gadalla A M, Bozkurt B and Faulk N M 1991 J. Am. Ceram. Soc. 74801

Ho K H and Newman S T 2003 Int. J. Mach. Tools Manuf. 43 1287

Norasetthekul S, Eubank P T, Bradley W L, Bozkurt B and Stucker B 1999 J. Mater. Sci. 341261

Prasad Y V R K, Sastry D H and Deevi S C 2001 Mater. Sci. \& Engg. A311 42

Singh S, Maheshwari S and Pandy P C 2004 J. Mater. Proc. Technol. 149272

Yamaguchi M, Inui H and Ito K 2000 Acta Mater. 48307 